\title{
The Inductive Coupling of the Magnets in MICE and its Effect on Quench Protection*
}

\author{
Michael A. Green ${ }^{1}$, Holger Witte ${ }^{2}$, and Bruce Strauss ${ }^{3}$ \\ 1. Lawrence Berkeley National Laboratory, Berkeley CA 94703, USA \\ 2. Oxford University Physics Department, Oxford OX1-3PU, UK \\ 3. Office of Science, US Department of Energy, Washington DC 20010, USA
}

September 2005

Presented at the 2005 Magnet Technology Conference (MT-19)

Genoa, Italy, 18 through 23 September 2005

Published in IEEE Transactions on Applied Superconductivity 16, No. 1 (2006)

\footnotetext{
* This work was supported by the Oxford University Physics Department and the Particle Physics and Astronomy Research Council of the United Kingdom. This work was also performed at the Lawrence Berkeley National Laboratory with the support of the Office of High Energy Physics, United States Department of Energy under DOE contract DE-AC02-05CH11231. DOE funding for the US Neutrino Factory and Muon Collider Collaboration is gratefully acknowledged.
} 


\section{DISCLAIMER}

This document was prepared as an account of work sponsored by the United States Government.

While this document is believed to contain correct information, neither the United States Government nor any agency thereof, nor The Regents of the University of California, nor any of their employees, makes any warranty, express or implied, or assumes any legal responsibility for the accuracy, completeness, or usefulness of any information, apparatus, product, or process disclosed, or represents that its use would not infringe privately owned rights. Reference herein to any specific commercial product, process, or service by its trade name, trademark, manufacturer, or otherwise, does not necessarily constitute or imply its endorsement, recommendation, or favoring by the United States Government or any agency thereof, or The Regents of the University of California. The views and opinions of authors expressed herein do not necessarily state or reflect those of the United States Government or any agency thereof, or The Regents of the University of California. 


\title{
The Inductive Coupling of the Magnets in MICE and its Effect on Quench Protection*
}

\author{
M. A. Green, H. Witte, and B. Strauss
}

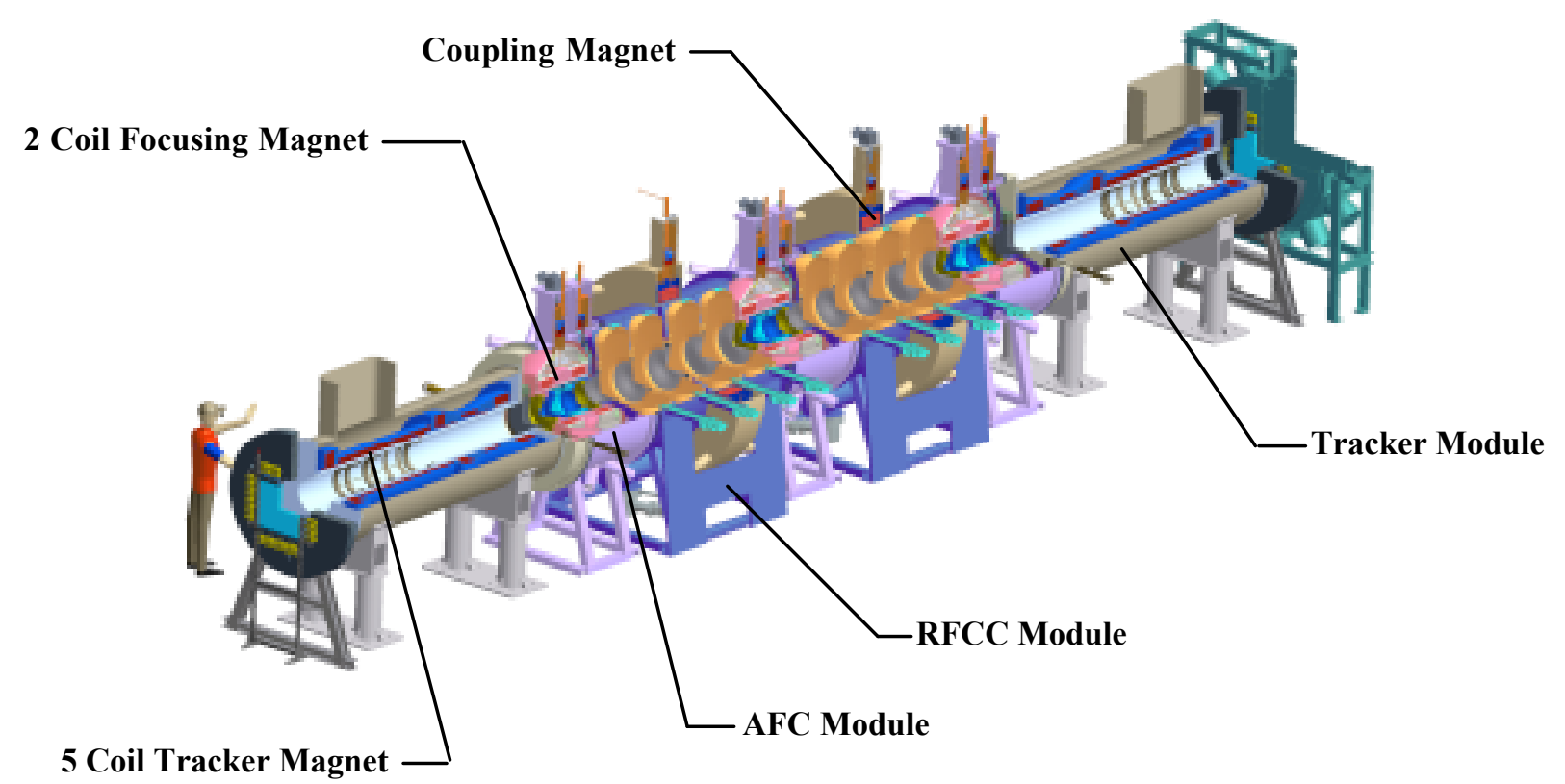

Fig. 1. The MICE Cooling channel with the two tracker magnets. The 18-coil system is coupled together magnetically.

\begin{abstract}
The inductive coupling between various MICE magnet circuits is described. The consequences of this coupling on magnet charging and quenching are discussed. Magnet quench protection is achieved through the use of quench-back. Calculations of the quenching of a magnet due to quench-back resulting from circulating currents induced in the magnet mandrel due to quenching of an adjacent magnet are discussed. This report describes how the MICE magnet channel will react when magnets in that channel are quenched.
\end{abstract}

Index Terms-S/C Solenoids, and Inductive Coupling

\section{INTRODUCTION TO MICE}

$\mathrm{T}$ he development of a muon collider or a neutrino factory requires that beams of low emittance muons be produced. A key to the production of low emittance muons is muon cooling. A demonstration of muon cooling is essential to the development of muon accelerators and storage rings [1], [2]. The international Muon Ionization Cooling Experiment (MICE) will be a demonstration of muon cooling in a configuration of superconducting magnets [3] that may be useful for a neutrino factory.

Manuscript received 19 September 2005. M. A. Green is from the Lawrence Berkeley Laboratory, Berkeley CA 94729, USA H. Witte is from Oxford University Physics Department, Oxford OX1-3PU, UK. B. Struass is from the Office of High Energy Physics, US DOE, Washington DC 20585-1290 *This work was supported by the Oxford University Physics Department and PPARC of the UK and at the Lawrence Berkeley National Laboratory with the support of the Office of High Energy Physics, US Department of Energy under contract DE-AC02-05CH11231
The full MICE channel is shown in Fig. 1. The experiment consists of two tracker modules at the ends of a muon-cooling channel. The cooling channel consists of three absorber focus coil modules (AFC modules) and two RF coupling coil modules (RFCC modules).

\section{THE MICE MAGNETS}

The tracker module consists of a five-coil superconducting magnet [4]. The three spectrometer coils (End 1, Center, and End 2) are used to generate a uniform $4 \mathrm{~T}$ field in a $1-\mathrm{m}$ long region that is $300-\mathrm{mm}$ in diameter. The uniform field region contains five planes of scintillating fibers used to measure the muon emittance. There are two match coils match 1 and match 2 in the tracker solenoid that are used to match the beam into the uniform field region. Table 1 presents the tracker magnet coil parameters.

Ionization cooling of muons means that muons have their momentum reduced in both the longitudinal direction and the transverse direction by passing them through a low $Z$ absorber. (Liquid hydrogen is best.) The absorber is located within the warm bore of a two-coil superconducting solenoid that is part of the AFC module [5]. The purpose of the magnet is to focus the muon beam so that it has low $\square$. The focusing magnet parameters are shown in Table 2.

RF cavities are used to re-accelerate the muons to their original longitudinal momentum. These cavities are surrounded by the superconducting coupling solenoid [6]. The coupling coil parameters are shown in Table 3 . 
TABLE 1. THE NOMINAL DESIGN PARMAMETERS FOR THE MICE DETECTOR SOLENOID COILS

\begin{tabular}{lccccc}
\multicolumn{1}{c}{ Parameter } & Match 1 & Match 2 & End 1 & Center & End 2 \\
\hline Coil length (mm) & 198 & 197 & 110 & 1294 & 110 \\
Coil inner radius (mm) & 258 & 258 & 258 & 258 & 258 \\
Coil thickness (mm) & 46.2 & 26.4 & 61.6 & 24.2 & 68.2 \\
Number of layers & 42 & 24 & 56 & 22 & 62 \\
Number of turns per layer & 120 & 119 & 66 & 784 & 66 \\
Coil overall current density $\left(\mathrm{A} \mathrm{mm}^{-2}\right)$ & 147.6 & 161.3 & 136.8 & 146.9 & 145.4 \\
Coil current (A) & 267.8 & 293.8 & 249.5 & 265.9 & 265.2 \\
Coil self inductance (H) & 12.8 & 4.3 & $9.6^{*}$ & $41.6^{*}$ & $11.4^{*}$ \\
Coil Stored Energy at Current above (MJ) & 0.47 & 0.20 & 0.30 & 1.49 & 0.40 \\
\hline
\end{tabular}

* Note the self-inductance of the 3 spectrometer-coils connected in series is $78 \mathrm{H}$. At $266 \mathrm{~A}$, the stored energy is $2.8 \mathrm{MJ}$.

TABLE 2.

THE BASIC PARAMETERS OF THE FOCUSING MAGNET

\begin{tabular}{|c|c|c|}
\hline Parameter & Non-flip & Flip \\
\hline Coil separation (mm) & \multicolumn{2}{|c|}{200} \\
\hline Coil length (mm) & \multicolumn{2}{|c|}{210} \\
\hline Coil inner Radius (mm) & \multicolumn{2}{|c|}{263} \\
\hline Coil thickness (mm) & \multicolumn{2}{|c|}{84} \\
\hline Number of layers & \multicolumn{2}{|c|}{76} \\
\hline Number of turns per layer & \multicolumn{2}{|c|}{127} \\
\hline Magnet $J\left(\mathrm{~A} \mathrm{~mm}^{-2}\right)^{*}$ & 71.96 & 138.2 \\
\hline Magnet Current (A)* & 130.5 & 250.7 \\
\hline Magnet Self Inductance (H) & 137.4 & 98.6 \\
\hline Magnet Stored Energy $(\mathrm{MJ})^{*}$ & 1.17 & 3.10 \\
\hline
\end{tabular}

* Worst case values based on $p=240 \mathrm{MeV} / \mathrm{c}$ and $\square=420 \mathrm{~mm}$

TABLE 3.

DESIGN PARAMETERS FOR THE COUPLING MAGNET

Parameter

\begin{tabular}{lc}
\hline \hline Coil Length (mm) & 250 \\
Coil Inner Radius (mm) & 725 \\
Coil Thickness (mm) & 116 \\
Number of Layers & 104 \\
No. Turns per Layer & 151 \\
Magnet $\left.J(\mathrm{~A} \mathrm{~mm})^{-2}\right)^{*}$ & 115.5 \\
Magnet Current (A)* & 213.2 \\
Magnet Self Inductance (H) & 563 \\
Magnet Stored Energy (MJ)* & 13.6
\end{tabular}

* Worst case design based on $p=240 \mathrm{MeV} / \mathrm{c}$ and $\square=420 \mathrm{~mm}$

Table 2 shows two operating modes for the focusing magnet, the flip mode where the field polarity changes as one goes through the magnet center along the magnet axis and the non-flip mode where the field polarity doesn't change. When MICE is run in the flip mode, the field polarity changes three times along the channel. In the non-flip mode there is no polarity change. MICE will be operated in both modes. The cases shown in Table 2 represent the worst cases for MICE.
The entire MICE channel (including the tracker module) consists of eighteen superconducting coils that are located within the seven cryostats. All eighteen coils are coupled with each other. The greatest coupling will be with adjacent coils. A quench of one magnet can affect other magnets.

\section{MICE MAGNET QUENCHES}

The primary mode of quench protection for the MICE focusing magnet is through quench-back from the 6061-T6aluminum mandrel that is around the two coils. Each focusing magnet consists of two coils hooked in series either in the flip mode or the non-flip mode depending on the experiment operating-configuration. Fig. 2 shows the current decay and the coil hot spot temperature as a function of the time from the start of a magnet quench. Fig. 2 applies for the focusing magnet hooked up in the worst-case flip mode (see Table 2). The two cases shown are for a quench of a single focusing magnet and a quench of the three magnets hooked in series. From Fig. 2, one can see that the three magnets quench is similar a single magnet. The quench-back time is short $(\sim 1.1 \mathrm{~s})$ so the energy is more evenly spread between magnets. Thus connecting the three focusing magnets together in series appears to be attractive [7], [8]. At the end of the quench the mandrel temperature is a lot cooler than the average coil temperature ( $52 \mathrm{~K}$ versus $82 \mathrm{~K}$ ).

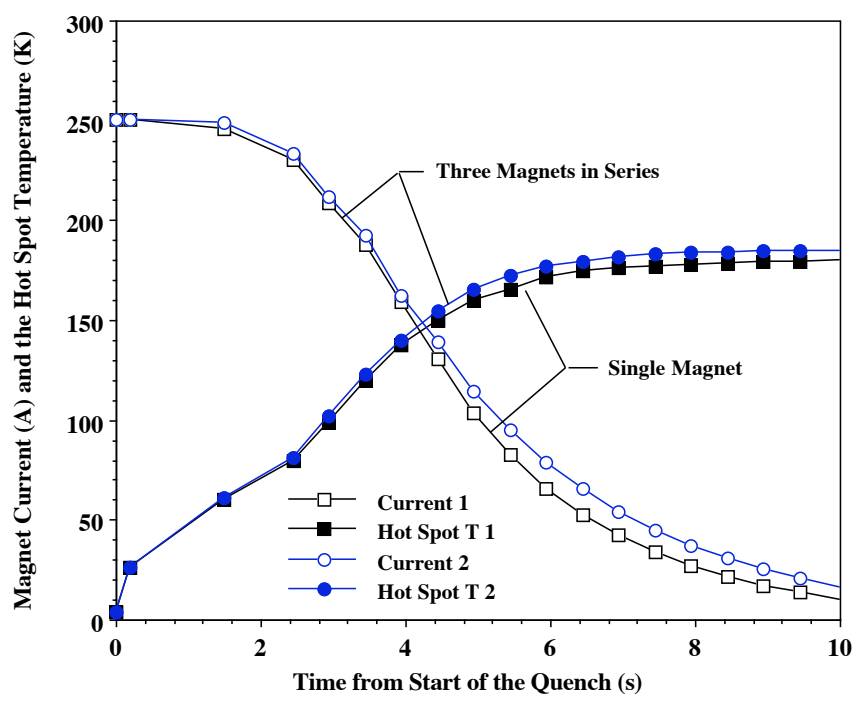

Fig 2. A quench of the one and three focusing magnets. 
TABLE 4. THE INDUCTANCE MAP FOR THE MICE MAGNET CIRCUITS IN THE FLIP MODE

\begin{tabular}{ccccccc} 
& S & M2 & M1 & F & C1 & C2 \\
\hline \hline S & 155.8 & 1.283 & 0.711 & 0.190 & 0.549 & 0.549 \\
M2 & 1.283 & 6.880 & 0.809 & 0.121 & 0.132 & 0.132 \\
M1 & 0.711 & 0.809 & 26.24 & 1.160 & 0.441 & 0.441 \\
F & 0.190 & 0.121 & 1.160 & 304.4 & 5.569 & 5.569 \\
C1 & 0.549 & 0.132 & 0.441 & 5.569 & 563.0 & 6.713 \\
C2 & 0.549 & 0.132 & 0.441 & 5.569 & 6.713 & 563.0 \\
\hline
\end{tabular}

TABLE 5. THE INDUCTANCE MAP FOR THE MICE MAGNET CIRCUITS IN THE NON-FLIP MODE

\begin{tabular}{ccccccc} 
& S & M2 & M1 & F & C1 & C2 \\
\hline \hline S & 156.5 & 1.285 & 0.721 & 0.705 & 0.810 & 0.810 \\
M2 & 1.285 & 6.886 & 0.809 & 0.278 & 0.161 & 0.161 \\
M1 & 0.721 & 0.809 & 26.46 & 1.963 & 0.631 & 0.631 \\
F & 0.705 & 0.278 & 1.963 & 416.3 & 17.91 & 17.91 \\
C1 & 0.810 & 0.161 & 0.631 & 17.91 & 563.0 & 6.713 \\
C2 & 0.810 & 0.161 & 0.631 & 17.91 & 6.713 & 563.0 \\
\hline
\end{tabular}

Similar quench studies were done on the coupling magnet (see Table 3) [7], [9]. Figure 3 shows the current decay of the coupling magnet and the coil hot-spot temperature as a function of the time from the quench start. The quench was simulated for a single coupling magnet and for both magnets in series. The results of the quench study suggest that the two magnets could be connected in series, but the hot-spot temperature is high. The quench back time is longer $(\sim 2.2 \mathrm{~s})$ for the coupling magnet. In this case the average coil temperature about the same as the mandrel (98 versus $96 \mathrm{~K}$ ).

Fig. 2 shows that three focusing magnets in series $\mathbf{F}$ will have an average $d i / d t=40 \mathrm{~A} \mathrm{~s}^{-1}$ during a quench. A single coupling magnet $\mathbf{C 1}$ or $\mathbf{C 2}$ will have a $d i / d t=25 \mathrm{~A} \mathrm{~s}^{-1}$ when it quenches. When the two spectrometer magnets $\mathbf{S}$ are hooked in series, di/dt of magnet circuit $\mathbf{S}$ is $\sim 50 \mathrm{~A} \mathrm{~s}^{-1}$. The match coils M1 and M2 will be connected in series with the corresponding coils in the tracker module at the opposite end of MICE. The average $d i / d t$ values for circuits M1 and M2 are about $60 \mathrm{~A} \mathrm{~s}^{-1}$ and $70 \mathrm{~A} \mathrm{~s}^{-1}$, respectively.

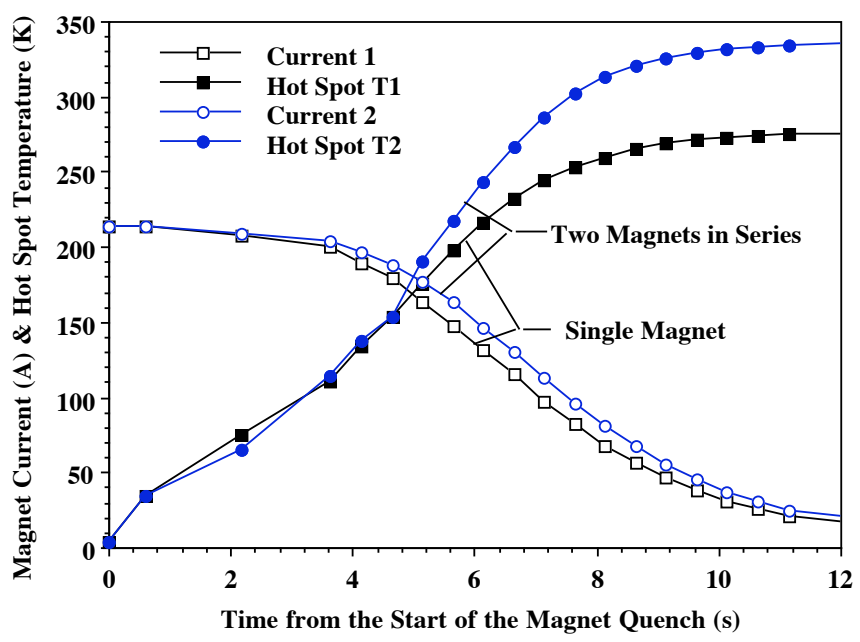

Fig 3. A quench of the one and two coupling magnets.

\section{COUPLING BETWEEN MICE MAGNETS}

Table 4 shows an inductance map for the six MICE magnet circuits S, M1, M2, F, C1 and C2 when MICE is operated in the flip mode. Table 5 shows an inductance map for all of the MICE magnet circuits when MICE is operated in the non-flip mode. The diagonal from the upper left hand corner to the lower right hand corner shows the self-inductance of each magnet circuit. The off-diagonal terms are the mutual inductances between the various MICE magnet circuits. One can see that the mutual inductance is highest between a magnet circuit and its nearest neighbor magnet circuits.

Using the mutual inductance $M_{1-2}$ between two magnet circuits, one can determine the induced voltage $V_{2}$ in the second magnet circuit due to a rate of current change $d i_{1} / d t$ in a first magnet circuit. An expression for this is given as follows:

$$
V_{2}=\square M_{1 \square 2} \frac{d i_{1}}{d t}
$$

The primary power supplies for the MICE magnet circuits generate $\pm 10 \mathrm{~V}$ with a maximum current of $300 \mathrm{~A}$ [10]. During charging, the $d i / d t$ in a circuit does not induce enough voltage in other magnet circuits to affect the charging very much. The power supply produces enough voltage to counter the induced voltage from other circuits.

For example a worst-case quench of the focusing magnets can induce $223 \mathrm{~V}$ in a coupling magnet circuit when operating in the flip mode and $716 \mathrm{~V}$ when operating in the non-flip mode. The $d i / d t$ in the coupling coil due to these voltages is $0.40 \mathrm{~A} \mathrm{~s}^{-1}$ and $1.27 \mathrm{~A} \mathrm{~s}^{-1}$ respectively for about five seconds. The rate of current change is unlikely to drive the coupling magnet normal through conductor AC loss or by driving the magnet to its critical current. Enough energy may be put into the mandrel to cause the magnet to quench through quench-back. Whether or not a quench in one magnet induces a quench in another magnet through quenchback from the magnet mandrel depends on the available temperature margin in the magnet. 
The focusing coil will also affect the first match coil in the tracker module. A worst-case quench of the focusing magnet will induce about $46 \mathrm{~V}$ in the match coil in the flip mode and about $79 \mathrm{~V}$ in the non-flip mode. The $d i / d t$ in the first match coil will be $1.74 \mathrm{~A} \mathrm{~s}^{-1}$ and $2.99 \mathrm{~A} \mathrm{~s}^{-1}$, respectively. As with the coupling coil, neither case is likely to result in a quench due to conductor $\mathrm{AC}$ loss or the coil being driven to its critical current. Quench-back in the mandrel can potentially cause quenching in the first match coil, although a quench in a focusing magnet is less likely to induce a quench in the first match coil than it is in the coupling coil because the temperature margin is higher for the entire tracker magnet system.

A quench in one magnet can cause quenching in the whole MICE channel, because the coil circuits are coupled together inductively. A quench in one magnet circuit can induce a quench in an adjacent magnet circuit due to quench-back. A quench in a second circuit is likely to induce a quench in a third circuit and so on, until the whole channel has quenched. The frequency of quenches in MICE will probably determine the acceptable range of average momenta of the muons used in the MICE channel. (The lower the average muon momentum, the lower the currents in the magnets for a given beam $\square$ in the center of the focusing magnet.)

\section{CONCLUding COMMEnTS}

The focusing magnets, coupling magnets and tracker magnets are designed so that they will quench passively without a quench detection system and an active quench protection system. The passive quench protection uses quench-back from the magnet mandrel to the to the magnet coils to spread the quench throughout the magnet coils.

The MICE coils will be connected together in six primary magnet circuits. All three focusing magnets will form one circuit. The coupling coils will be individually powered (two more circuits). Match coil M1 and M2 will each be connected in series with its corresponding coil in the other tracker magnet. The spectrometer solenoid of one tracker magnet will be connected in series with the spectrometer magnet in the other tracker magnet. Tuning of the end coils on each spectrometer magnet will be done using small power supplies. The small tuning supplies have a small effect on the overall magnetic coupling within MICE.

Inductance networks were calculated for the six MICE primary magnet circuits for operation in both the flip and non-flip modes for MICE. As a result of the proximity of the coils and the proposed method of connecting the magnets electrically, every coil in MICE is coupled magnetically with every other coil in MICE. The magnet coupling will affect magnet charging minimally because there is enough voltage capacity in the power supplies to counter the voltage induced by the charging of an adjacent coil circuit.

A quench of one magnet circuit is unlikely to induce a quench in another magnet circuit through $\mathrm{AC}$ losses in the conductor or through driving the magnet current beyond its crucial current. A quench in one magnet circuit may cause an adjacent magnet circuit to quench through quench-back from the magnet mandrel. Whether a quench in one MICE magnet circuit causes another magnet circuit to quench depends on the temperature margin of the coils being quenched by the second magnet circuit.

\section{ACKNOWLEDGEMENTS}

The authors acknowledge the work of James Rochford and Yury Ivanyushenkov of the Rutherford Appleton Laboratory, for checking much of the data that went into this report.

\section{REFERENCES}

[1] N. Holtkamp and D. Finley Eds, "A Feasibility Study of a Neutrino Source Based on a Muon Storage Ring," FERMI-Pub-00/108E, (2000).

[2] R. B. Palmer, A. Sessler, A. Skrinsky, A. Tollestrup, et al, "Muon Colliders, " Brookhaven National Laboratory Report BNL-62740, January 1996.

[3] "MICE and International Muon Ionization Cooling Experiment Technical Reference Document," co-authored with G. Gregoire, G. Ryckewaert, L. Chevalier, et al, http://hep04.phys.itt.edu/cooldemo, (October 2004)

[4] P. Fabricatore, S. Farinon, U. Bravar, and M. A. Green, "The Mechanical and Thermal Design for the MICE Detector Solenoid Magnet System," IEEE Transactions on Applied Superconductivity 15, No. 2, p 1279, (2005).

[5] S. Q. Yang, M. A. Green, G. Barr, et al, "The Mechanical and Thermal Design for the MICE Focusing Solenoid Magnet System," IEEE Transactions on Applied Superconductivity 15, No. 2, p 1259, (2005),

[6] M. A. Green, S. Q. Yang, U. Bravar, et al, "The Mechanical and Thermal Design for the MICE Coupling Solenoid Magnet," IEEE Transactions on Applied Superconductivity 15, No. 2, p1255, (2005).

[7] M. A. Green and H. Witte, "Quench Protection and Magnet Power Supply Requirements For the MICE Focusing and Coupling Magnets," MICE Note 114, http://hep04.phys.itt.edu/cooldemo, (June 2005).

[8] M. A. Green, Y, Ivanyushenkov, W. Lau, et al, "Progress on the Focus Coils for the MICE Channel." Proceedings of the 2005 Particle Accelerator Conference, Knoxville TN, 16 to 20 May 2005, MICE Note $118 \mathrm{http}: / /$ hep04.phys.itt.edu/cooldemo, (May 2005).

[9] M. A. Green, D. Li, S. Virostek, and H. Witte, "Progress on the Coupling Coil for the MICE Channel," Proceedings of the 2005 Particle Accelerator Conference, Knoxville TN, 16 to 20 May 2005, MICE Note 117 http://hep04.phys.itt.edu/cooldemo, (May 2005).

[10] M. A. Green and H. Witte, "Power Supply and Quench Protection for the MICE Cooling Channel Magnets" to be published in Journal of Physics Conference Series with other papers from EUCAS-2005, Vienna Austria, 11 to 14 September 2005. 